\title{
Robust Controller for Vertical-Longitudinal-Lateral Dynamics Control of Small Helicopter
}

\author{
Tushar K. Roy \\ Department of ETE, RUET, Bangladesh \\ *Corresponding Author: roy_kanti@yahoo.com
}

Copyright (C) 2014 Horizon Research Publishing All rights reserved.

\begin{abstract}
The problem of stabilization of vertical, lateral and longitudinal dynamics for hover flight mode of an unmanned autonomous helicopter (UAH) in a gusty environment is considered. The controller design is based on a generic linear model which successfully describes the behavior of most small scale helicopters. A recursive (backstepping) design procedure is used to design the robust controller for vertical, longitudinal and lateral dynamics based on the Lyapunov's method. For comparison purposes we design another controller based on the linear quadratic regulator (LQR) criteria. The simulation results demonstrate that the proposed controllers can effectively attenuate the gust effects and achieve rapid and accurate vertical, longitudinal and lateral position tracking when gusts occur.
\end{abstract}

Keywords Robust Backstepping Controller, LQR, External Wind Gusts, Lyapunov Function

\section{Introduction}

Among the variety of Unmanned Air Vehicles (UAVs), unmanned autonomous helicopters constitute one of the most versatile and agile platforms for the development of autonomous flight systems. A helicopter can operate in different flight modes, such as vertical take-off/landing, hovering, longitudinal/lateral flight, and bank to turn which gives them the advantage of effective observation from various positions. Among these abilities hovering and vertical take off ability are necessarily needed. And due to their high level of agility, maneuverability and capability of operating in adverse weather conditions there are new trends of unmanned autonomous helicopter controller design nowadays. So, unmanned autonomous helicopter control system should make these performances achieved by improving the tracking performance and disturbance rejection capability. Robustness is one of the critical issues which must be considered in the control system design for small unmanned autonomous helicopter, especially those covering large flight envelope. One major problem rarely addressed by researchers to date is that of a wind disturbance.
To cope with such a problem researchers have considered approaches such as robust [9], neural network [10] etc. In this paper, we propose two controllers to stabilize the vertical, longitudinal and lateral dynamics of the small scale helicopter in the presence of wind gusts. A robust $\mathrm{H}_{\infty}$ control method of the longitudinal and lateral dynamics of the BELL 205 helicopters in the presence of model uncertainty is presented in [1]. A robust feedback method for helicopter stabilization to reject wind disturbance is presented in [2], wherein the wind disturbance is assumed to be the sum of a fixed number of sinusoids with unknown amplitudes, frequencies and phases. In [3], the authors present a robust backstepping technique of an autonomous scale helicopter subject to parameters uncertainties and uniform time varying tridimensional wind gusts. With the assistance of an unknown input observer technique (UIO), the controller is reported to be able to handle the effect of these uncertainties on the autonomous helicopter. In [4] the authors propose a nonlinear $\mathrm{H}_{\infty}$ horizontal position controller for hover and automatic landings of a RUAV in the presence of horizontal wind gusts. Control of a model scale helicopter under vertical wind gusts is discussed in [5]. The typical level of wind gust in this paper is less than $1 \mathrm{~m} / \mathrm{s}$. Furthermore, the authors present an active disturbance rejection control strategy based on a nonlinear observer. In [12], Ho robust controller for altitude and attitude tracking are designed for hovering flight in the presence of vertical wind gusts and they added the vertical wind gusts with the vertical speed but they did not mention how to control the lateral and longitudinal position. In the paper of [13] the authors presented an application of nonlinear robust regulation and nonlinear small-gain methods to control the vertical motion problem of an autopilot for helicopters landing under parameter uncertainties on the plant and actuator model. For hover control of a helicopter, the work in [14] introduced a preliminary study on the use of neural adaptive control techniques by considering a simple one Degree of Freedom (1-DOF) system. In the previous version of this paper [15], the authors designed a robust backstepping controller for horizontal position control of a small helicopter by considering the effect of external wind gusts. However, in our case we concentrate on the longitudinal, lateral and 
vertical external uncertainties at the same time. The main control purpose addressed here is to design the three control inputs in order to asymptotically track vertical, lateral and longitudinal references $z_{\text {ref }}(t), y_{\text {ref }}(t)$, and $x_{\text {ref }}(t)$, respectively. So, this model consists of three parts with different physical backgrounds: A first subsystem describes vertical motions which are controlled by the collective pitch of the main rotor. It is strongly coupled with the torque interaction between the main rotor and the fuselage. On the other hand, forward and sideward motions, together with roll and pitch motions are controlled by lateral and longitudinal cyclic stick inputs via the flapping motion of the main rotor (second and third subsystems).

Taken into account such external wind disturbances in the model equations, we use Lyapunov's approach to show that our proposed backstepping controller is robust with respect to external wind gusts. The performances of the controllers are simulated and tested in keeping the positions constantly in the presence of external wind gusts. The rest of this paper is organized as follows. Section (II) briefly introduces the mathematical model used and Section (III) introduces the gusts model. Section (IV) presents the control structure. Section (V) discusses simulation results. Finally, the paper concludes in Section (VI).

\section{Helicopter Linear Model}

Among other UAVs, Unmanned Autonomous Helicopter (UAH) has the specific characteristic; the helicopter can move vertically, float in the air, turn in place, move forward and laterally and can perform these movements in combinations. Because of this, helicopter dynamic modeling is a very complex problem. In $6 \mathrm{DOF}$ form, the motion states and control inputs are represented as

$$
\begin{aligned}
& x=\{u, w, q, \theta, v, p, r, \phi\} \\
& u_{c}=\left\{\delta_{c o l}, \delta_{\text {lat }}, \delta_{\text {lat }}, \delta_{\text {ped }}\right\}
\end{aligned}
$$

The variables $u, v$ and $w$ represent the helicopter linear velocity in body frame; $p, q$ and $r$ denote roll, pitch and yaw rates respectively; and $\phi, \theta$ represent roll, pitch attitude respectively. A conventional single main rotor helicopter has four independent control inputs, $\delta_{\text {lat }}, \delta_{\text {lon }}, \delta_{c o l}$ and $\delta_{\text {ped }}$ which denote the deflection of the lateral cyclic, longitudinal cyclic, main rotor collective pitch and tail rotor collective pitch respectively. The collective commands control the magnitude of the main rotor and tail rotor thrust and other two control commands control the inclination of the Tip-Path-Plane (TPP) on the longitudinal and lateral direction.

Before getting into the control law design longitudinal and lateral dynamics should be analyzed. For this purpose, the nonlinear form of a helicopter equations of motion are given as follows,

$$
\dot{u}=(v r-w q)-g \sin \theta+X / m
$$

$$
\begin{gathered}
\dot{v}=(w p-u r)+g \sin \phi \cos \theta+Y / m \\
\dot{w}=(u q-v p)+g \cos \phi \cos \theta+Z / m \\
\dot{p}=q r\left(I_{y y}-I_{z z}\right) / I_{x x}+L / I_{x x} \\
\dot{q}=p r\left(I_{z z}-I_{x x}\right) / I_{y y}+M / I_{y y} \\
\dot{r}=p q\left(I_{x x}-I_{y y}\right) / I_{z z}+N / I_{z z}
\end{gathered}
$$

where, where, forces $[\mathrm{X}, \mathrm{Y}, \mathrm{Z}]^{\prime}$ and moments $[\mathrm{L}, \mathrm{M}, \mathrm{N}]$ ' are expressed in the body frame, $m$ is the helicopter mass and $\mathrm{I}_{\mathrm{xx}}$, $\mathrm{I}_{\mathrm{yy}}$ and $\mathrm{I}_{\mathrm{zz}}$ are the moment of inertial about $\mathrm{x}, \mathrm{y}$, and $\mathrm{z}$ axis. In order to complete the system, we need to the following two equations which relate the Euler angle rates to the angular velocity [7].

$$
\begin{gathered}
\dot{\phi}=p+q \sin \phi \tan \theta+r \cos \phi \tan \theta \\
\dot{\theta}=q \cos \phi-r \sin \phi
\end{gathered}
$$

We know that the cyclic longitudinal and lateral tilt of the main rotor disk is controllable through the cyclic pitch. Therefore, in this model, the longitudinal and lateral flapping dynamics can be represented by the first order equations as follows [8]:

$$
\begin{aligned}
& \dot{a}_{1}=-q-\frac{a_{1}}{\tau}+\frac{1}{\tau}\left(\frac{\partial a}{\partial u} u+A_{\text {lon }} \delta_{l o n}\right) \\
& \dot{b}_{1}=-p-\frac{b_{1}}{\tau}+\frac{1}{\tau}\left(\frac{\partial b}{\partial u} v+A_{\text {lat }} \delta_{\text {lat }}\right)
\end{aligned}
$$

where, $\delta_{l a t}$ and $\delta_{\text {lon }}$ are the lateral and longitudinal cyclic control inputs, $a_{1}$ and $b_{1}$ are the lateral and longitudinal flapping angles and $A_{\text {lon }}$ and $B_{\text {lat }}$ are effective steady-state longitudinal and lateral gains from the cyclic inputs to the main rotor flapping angles. In Eqn. (9) and Eqn. (10), $A_{u}=\frac{\partial a_{1}}{\partial u}$ and $B_{v}=\frac{\partial b_{1}}{\partial v}$ are constants and represent the longitudinal and lateral Dihedral effect. The dihedral effect is the change of tip-path-plane (TPP) tilt due to the longitudinal and lateral velocities [16]. The Dihedral effect is modeled by the following equation

$$
\frac{\partial a_{1}}{\partial u}=-\frac{\partial b_{1}}{\partial v}=\frac{2}{\Omega R_{b}}\left(\frac{8 C_{T}}{a \sigma}+\sqrt{\frac{C_{T}}{2}}\right)
$$

where, $R_{b}$ is the main rotor radius, $\sigma$ solidity ratio, 'a' lift curve slope and $C_{T}$ thrust coefficient. Since the rotor is symmetric, we consider $A_{u}=-B_{v}$.

\section{A. Formation of Lateral and Longitudinal Dynamics}


Linearization is essential to derive simplified working models, considering inherent instability under hover and slow flight conditions. So, after linearizing equations (Eq. 1-8) we get following parameterized model of decoupled longitudinal and lateral dynamics.

$$
\begin{aligned}
& {\left[\begin{array}{c}
\dot{u} \\
\dot{q} \\
\dot{\theta} \\
\dot{a}_{1}
\end{array}\right]=\left[\begin{array}{cccc}
X_{u} & X_{q} & -g & X_{a} \\
M_{u} & M_{q} & 0 & M_{a} \\
0 & 1 & 0 & 0 \\
\frac{A_{u}}{\tau} & -1 & 0 & -\frac{1}{\tau}
\end{array}\right]\left[\begin{array}{c}
u \\
q \\
\theta \\
a_{1}
\end{array}\right]+\left[\begin{array}{c}
0 \\
0 \\
0 \\
\frac{A_{\text {lon }}}{\tau}
\end{array}\right] \delta_{\text {lon }}} \\
& {\left[\begin{array}{c}
\dot{v} \\
\dot{p} \\
\dot{\phi} \\
\dot{b_{1}}
\end{array}\right]=\left[\begin{array}{cccc}
Y_{v} & Y_{p} & g & Y_{b} \\
L_{v} & L_{p} & 0 & L_{b} \\
0 & 1 & 0 & 0 \\
\frac{B_{v}}{\tau} & -1 & 0 & -\frac{1}{\tau}
\end{array}\right]\left[\begin{array}{c}
v \\
p \\
\phi \\
b_{1}
\end{array}\right]+\left[\begin{array}{c}
0 \\
0 \\
0 \\
\frac{A_{\text {lat }}}{\tau}
\end{array}\right] \delta_{\text {lat }}}
\end{aligned}
$$

where $X_{u}=\frac{1}{m} \frac{\partial X}{\partial u}, M_{u}=\frac{1}{I_{y y}} \frac{\partial M}{\partial u} \ldots$ are the force and moment derivatives normalized by the mass of the helicopter or respective moment of inertia. The pitching flap-stiffness constant is represents by $M_{a}$ that can be computed as follows [17]: $M_{a}=\frac{m M_{z}}{I_{y y}}+\frac{K_{\beta}}{I_{y y}}$ where $M_{z}$ is the height of the rotor hub above the fuselage center of gravity, $\mathrm{I}_{\mathrm{yy}}$ is the pitching moment of inertia and $K_{\beta}$ is the rotor blade spring stiffness. Similarly the lateral flap-stiffness constant $L_{b}$ can be computed as follows: $L_{b}=\frac{m M_{z}}{I_{x x}}+\frac{K_{\beta}}{I_{x x}}$.

The proposed linear model has been successfully adopted for control applications in a large number of small-scale unmanned helicopters [18-26]. In order to stabilize and control the helicopter system for longitudinal and lateral dynamics, we use robust backstepping controller.

Remark 1: Control inputs in the controller design process are set to be longitudinal and lateral flapping angles. They will be converted later into longitudinal cyclic and lateral cyclic for implementation.

\section{B. Formation of Vertical Dynamics}

Since we are also interested to control the altitude so, we need to express the equation (3) i.e. $\mathrm{z}$ dynamics in earth frame. To this end, we use the rotation matrix between the body and earth frames and obtain the following altitude dynamics:

$$
\dot{w}=g+(\cos \phi \cos \theta) Z / m
$$

Altitude dynamics can be linearized around hover conditions, i.e., $\phi \approx 0, \theta \approx 0$, and $\psi \approx 0$. So, resulting linearized altitude dynamics can be expressed in the earth frame as

$$
\dot{w}=\frac{m g-T}{m}
$$

\section{Gust Model}

The purpose of including wind gusts into the simulation model is to ensure that the design controller can cope with a real-world environment where wind effects can be a significant challenge to station keeping. For analyses of RUAV, wind gusts can be treated as either random (spectral turbulence) or discrete. For random gusts, typical spectral models include the Von Karman and Dryden turbulence models. The Von Karman model has widely been considered the more "realistic" model when it comes to defining turbulence spectra. However, due to the computational complexity of the Von Karman model, the Dryden model is typically used in aerospace vehicle analyses. There are many sources for wind models based upon empirical data that consist of passing band limited white noise through appropriate forming filters. The turbulence models are scaled with respect to RUAV altitude, velocity, wing span. Vertical wind gusts can be neglected compared with its horizontal counterparts due to small quantity component. Consequently we consider the horizontal wind gusts model in this work, and corresponding forming filters including $H_{u}(s)$ for longitudinal direction and $H_{v}(s)$ for lateral direction, take the following transfer function forms, [11] respectively:

$$
\begin{array}{r}
H_{u}(s)=\sigma_{u} \sqrt{\frac{2 L_{u}}{\pi U}} \frac{1}{1+\frac{L_{u}}{U} s} \\
H_{v}(s)=\sigma_{v} \sqrt{\frac{L_{u}}{\pi U} \frac{1+\frac{\sqrt{3} L_{v}}{U} s}{\left(1+\frac{L_{v}}{U} s\right)^{2}}} \\
H_{w}(s)=\sigma_{v} \sqrt{\frac{L_{w}}{\pi U}} \frac{1+\frac{\sqrt{3} L_{w}}{U} s}{\left(1+\frac{L_{w}}{U} s\right)^{2}}
\end{array}
$$

where, $\mathrm{U}$ is the true speed of a RUAV, $\sigma_{u}$, and $\sigma_{v}$ are the root mean square intensities of the turbulence and $L_{u}$, and $L_{v}$ are the turbulence scale lengths that describe the behavior of the wind gusts. In this work, the scale of turbulence, $L_{u}$, and $L_{v}$ are assigned constant values of $\mathrm{L}_{\mathrm{u}}=L_{v}=722.5 \mathrm{~m}$. And for low altitude region (altitude $<1000 \mathrm{ft}$ ) the $\sigma_{u}, \sigma_{v}$, and $\sigma_{w}$ turbulence intensities are given by

$$
\sigma_{w}=0.1 W_{20}
$$




$$
\frac{\sigma_{u}}{\sigma_{w}}=\frac{\sigma_{v}}{\sigma_{w}}=\frac{1}{(0.177+0.000823 h)^{0.4}}
$$

where, $W_{20}$ is the wind speed at $20 \mathrm{ft}(6 \mathrm{~m})$ above the ground and can be approximated by $U$ and altitude is described by $h$. In this paper, we have considered a typical wind speed of 10 $\mathrm{m} / \mathrm{s}$ and an altitude of $2 \mathrm{~m}$.

\section{Controller Design}

In this section, we design the controller for the Vertical, longitudinal and lateral dynamics of the helicopter dynamics separately and each based on an appropriate decoupled model. And for that a robust backstepping control method-based on Lyapunov's function for the position stabilize of a small scale helicopter is presented through the control of longitudinal and lateral flapping angles and collective pitch as the control inputs. But for comparison purposes we also design another controller based on the linear quadratic regulator (LQR) criteria. The linear motion equations for longitudinal and lateral dynamics are described through Eq. (11-12). In the longitudinal dynamics (11), the parameter $a_{1}$ is a function of $u$ and $q$. Similarly in the lateral dynamics (12), the parameter $b_{1}$ is a function of $v$ and $p$. Hence, we cannot carry out the flight control laws via backstepping considering $a_{1}$ and $b_{1}$ as the control input. A common simplification practice, presented in [6] is to neglect the effect of the lateral and longitudinal forces produced by the flapping angles. These parasitic forces have a minimal effect on the translational dynamics compared to the propulsion forces produced by the stability derivatives $X_{\theta}$ and $X_{\phi}$ (in (11) and (12) are denoted by $-\mathrm{g}$ and $\mathrm{g}$, respectively). We also neglect the stability derivative terms $X_{q}$ and $Y_{p}$ for deriving controller, because they are much smaller than the propulsion forces produced by the stability derivatives $X_{\theta}$ and $X_{\phi}$ This assumption is physically meaningful and results into a linear system in feedback form.

According to our design purposes the helicopter should be separated into two interconnected subsystems. The first subsystem accounts for longitudinal mode and second subsystem accounts for lateral mode. As indicated in the above, the effect of the translational forces produced by the flapping motion of the main rotor is parasitic and negligible compared to the main source of propulsion, which are the forces produced by the roll and pitch attitude change of the fuselage. By neglecting the effect of the parameters $X_{a}, Y_{b}$, $X_{q}$ and $Y_{p}$ the longitudinal-lateral dynamics will have strict feedback forms. As a result the simplified description of the longitudinal mode is given as follows,

$$
\begin{array}{r}
\dot{x}=u, \dot{u}=X_{u} u-g \theta \\
\dot{\theta}=q, \dot{q}=M_{u} u+M_{q} q+M_{a} a_{1}
\end{array}
$$

Similarly the simplified description of the lateral mode is given as,

$$
\begin{aligned}
& \dot{y}=v, \dot{v}=Y_{v} v+g \phi \\
& \dot{\phi}=p, \dot{p}=L_{v} v+L_{p} p+L_{b} b_{1}
\end{aligned}
$$

\section{A. Longitudinal Dynamics}

In this subsection, the robust backstepping controller based on Lyapunov method is designed for the longitudinal dynamics in the presence of wind gusts. Linear model equations of the longitudinal dynamics under external disturbance are rewritten as:

$$
\begin{gathered}
\dot{x}=u, \dot{u}=X_{u} u-g \theta, \dot{\theta}=q \\
\dot{q}=M_{u} u+M_{q} q+M_{a} a_{1}+A
\end{gathered}
$$

where, $A$ is an unknown parameter which is estimated as $\hat{A}$. The estimation error on $A$ is assumed bounded by known constant $F$, that is, $\mid A-\hat{A} \leq F$

Step1: The design process starts with the definition of the longitudinal position error i.e.,

$$
z_{1}=x-x_{d}, \dot{z}_{1}=\dot{x}_{\mathrm{t}} \dot{x}_{d}, \quad \dot{z}_{1}=u
$$

We consider $u$ as a virtual control input and define $u_{d}$ as a virtual control law for Eqn. (17). Let $z_{2}$ be an error variable representing the difference between the actual and virtual control inputs i.e.,

$$
z_{2}=u-u_{d}, \quad u=z_{2}+u_{d}
$$

Therefore, . At this stage we would like to design a virtual control law ud which would make $z_{1} \rightarrow 0$ as $t \rightarrow \infty$ Consider a control Lyapunov function

$W_{1}=\frac{1}{2} z_{1}^{2}$

And its derivative as follows,

$$
\dot{W}_{1}=z_{1} \dot{z}_{1}, \quad \dot{W}_{1}=z_{1}\left(z_{2}+u_{d}\right)
$$

We can now select an appropriate virtual control law $u_{d}$ which would make $\dot{W}_{1} \leq 0$. A possible choice is $u_{d}=-k_{1} z_{1}$ then,

$$
\begin{array}{r}
\dot{W}_{1}=-k_{1} z_{1}^{2}+z_{1} z_{2} \text {. Clearly if } z_{2}=0 \text { then } \\
\dot{W}_{1}=-k_{1} z_{1}^{2} \leq 0 .
\end{array}
$$

where, $k_{l}$ is a scalar parameter which can be used to tune the output response. Now consider the time derivative of $u_{d}$ as

$$
\dot{u}_{d}=-k_{1} \dot{z}_{1}, \dot{u}_{d}=-k_{1} u
$$

Step2: We derive the error dynamics for $z_{2}=u-u_{d}$ and its time derivative as follows,

$$
\dot{z}_{2}=\dot{u}-\dot{u}_{d} \quad, \quad \dot{z}_{2}=x_{u} u-g \theta+k_{1} u
$$


In which $\theta$ is viewed as a virtual control input. Now define a virtual control law $\theta_{d}$ and let $z_{3}$ be an error variable representing the difference between actual and virtual controls i.e., $z_{3}=\theta-\theta_{d}$

$$
\dot{z}_{2}=\left(x_{u}+k_{1}\right) u-g\left(z_{3}+\theta_{d}\right)
$$

Now choose a control Lyapunov function as follows,

$W_{2}=W_{1}+\frac{1}{2} z_{2}{ }^{2}$. And its derivative as follows,

$$
\begin{aligned}
& \dot{W}_{2}=\dot{W}_{1}+z_{2} \dot{z}_{2} \\
& \dot{W}_{2}=-k_{1} z_{1}^{2}+z_{2}\left\{z_{1}+\left(x_{u}+k_{1}\right) u-g \theta_{d}\right\}-g z_{2} z_{3}
\end{aligned}
$$

We can now select an appropriate virtual control $\theta_{d}$ to cancel out some terms related to $z_{1}, z_{2}$ and $u$, while the term involving $\mathrm{z}_{3}$ cannot be removed.

$$
\theta_{d}=g^{-1}\left\{z_{1}+\left(x_{u}+k_{1}\right) u+k_{2} z_{2}\right\}
$$

Therefore, $\dot{W}_{2}=-k_{1} z_{1}^{2}-k_{2} z_{2}^{2}-g z_{2} z_{3}$.

Clearly if $z_{3}=0$ then $\dot{W}_{2}=-k_{1} z_{1}^{2}-k_{2} z_{2}^{2} \leq 0$.

Now the time derivative of $\theta_{d}$ is

$$
\begin{aligned}
& \dot{\theta}_{d}=g^{-1}\left\{\dot{z}_{1}+\left(x_{u}+k_{1}\right) \dot{u}+k_{2} \dot{z}_{2}\right\} \\
& \dot{\theta}_{d}=g^{-1}\left\{u+\left(x_{u}+k_{1}\right)\left(x_{u} u-g \theta\right)+k_{2} \dot{z}_{2}\right\}
\end{aligned}
$$

Step3: We derive the error dynamics for $z_{3}=\theta-\theta_{d}$.

And its time derivative as follows, $\dot{z}_{3}=\dot{\theta}-\dot{\theta}_{d}$

$$
\begin{aligned}
& \dot{z}_{3}=q-g^{-1}\left\{u+\left(x_{u}+k_{1}\right)\left(x_{u} u-g \theta\right)\right\} \\
& -g^{-1} k_{2}\left\{\left(x_{u}+k_{1}\right) u-g\left(z_{3}+\theta_{d}\right)\right\}
\end{aligned}
$$

In which $q$ is viewed as a virtual control input. Now define a virtual control law $q_{d}$ and let $z_{4}$ be an error variable representing the difference between actual and virtual control input i.e., $z_{4}=q-q_{d}$

Therefore,

$$
\begin{aligned}
& \dot{z}_{3}=z_{4}+q_{d}-g^{-1}\left\{u+\left(x_{u}+k_{1}\right)\left(x_{u} u-g \theta\right)\right\} \\
& -g^{-1} k_{2}\left\{\left(x_{u}+k_{1}\right) u-g\left(z_{3}+\theta_{d}\right)\right\}
\end{aligned}
$$

Now choose a control Lyapunov function ,

$$
\begin{aligned}
& W_{3}=W_{2}+\frac{1}{2} z_{3}^{2} \text {. And its time derivative as follows, } \\
& \qquad \dot{W}_{3}=\dot{W}_{2}+z_{3} \dot{z}_{3} \\
& \dot{W}_{3}=-k_{1} z_{1}^{2}-k_{2} z_{2}{ }^{2}-g z_{3} z_{2}+z_{3}\left[z_{4}+q_{d}\right. \\
& -g^{-1}\left\{u+\left(x_{u}+k_{1}\right)\left(x_{u} u-g \theta\right)\right\}-g^{-1} k_{2}\left\{\left(x_{u}+k_{1}\right) u\right. \\
& \left.\left.-g\left(z_{3}+\theta_{d}\right)\right\}\right]
\end{aligned}
$$

We can now select an appropriate virtual control $q_{d}$ to cancel out some terms related to $z_{1}, z_{2}, z_{3}$ and $u$, while the term involving $z_{4}$ cannot be removed.

$$
\begin{aligned}
& q_{d}=g z_{2}+g^{-1}\left\{u+\left(x_{u}+k_{1}\right)\left(x_{u} u-g \theta\right)\right\} \\
& \left.+g^{-1} k_{2}\left\{\left(x_{u}+k_{1}\right) u-g\left(z_{3}+\theta_{d}\right)\right\}\right]-k_{3} z_{3}
\end{aligned}
$$

And its derivative as follows,

$$
\begin{aligned}
& \dot{q}_{d}=g \dot{z}_{2}+g^{-1}\left\{\dot{u}+\left(x_{u}+k_{1}\right)\left(x_{u} \dot{u}-g \dot{\theta}\right)\right\} \\
& \left.+g^{-1} k_{2}\left\{\left(x_{u}+k_{1}\right) \dot{u}-g\left(\dot{z}_{3}+\dot{\theta}_{d}\right)\right\}\right]-k_{3} \dot{z}_{3}
\end{aligned}
$$

Suppose, $\dot{q}_{d}=f\left(z_{2}, z_{3}, u, \theta, \theta_{d}\right)$

Therefore,

$$
\dot{W}_{3}=-k_{1} z_{1}^{2}-k_{2} z_{2}^{2}-k_{3} z_{3}^{2}+z_{3} z_{4}
$$

Clearly if

$$
z_{4}=0 \text { then } \dot{W}_{3}=-k_{1} z_{1}^{2}-k_{2} z_{2}^{2}-k_{3} z_{3}^{2} \leq 0
$$

Step4: We derive the error dynamics for $z_{4}=q-q_{d}$. And its derivative as follows,

$$
\begin{aligned}
& \dot{z}_{4}=\dot{q}-\dot{q}_{d}, \dot{z}_{4}=M_{u} u+M_{q} q+M_{a} a_{1}+A \\
& -f\left(z_{2}, z_{3}, u, \theta, \theta_{d}\right)
\end{aligned}
$$

In the above equation the actual control input appears. Our objective is to design the actual control input ' $a_{1}$ ' such that $z_{l}$, $z_{2}, z_{3}$, and $z_{4}$ converge to zero as $\mathrm{t} \rightarrow \infty$.

Choose a Lyapunov function $W_{4}$ as, $W_{4}=W_{3}+\frac{1}{2} z_{4}{ }^{2}$.

And its time derivative as follows,

$$
\begin{aligned}
& \dot{W}_{4}=\dot{W}_{3}+z_{4} \dot{z}_{4} \\
& \dot{W}_{4}=-k_{1} z_{1}^{2}-k_{2} z_{2}^{2}-k_{3} z_{3}^{2}+z_{3} z_{4}+ \\
& z_{4}\left(M_{u} u+M_{q} q+M_{a} a_{1}+A-f\left(z_{2}, z_{3}, u, \theta, \theta_{d}\right)\right)
\end{aligned}
$$

We are finally in the position to design control ' $a_{l}$ ' by making $\dot{W}_{4} \leq 0$ as follows,

$$
\begin{aligned}
& a_{1}=-M_{a}^{-1}\left(z_{3}+M_{u} u+M_{q} q+\hat{A}-\right. \\
& \left.f\left(z_{2}, z_{3}, u, \theta, \theta_{d}\right)+k_{4} z_{4}+F \operatorname{sgn}\left(z_{4}\right)\right)
\end{aligned}
$$

Therefore,

$$
\begin{aligned}
\dot{W}_{4}= & -k_{1} z_{1}^{2}-k_{2} z_{2}{ }^{2}-k_{3} z_{3}^{2}-k_{4} z_{4}{ }^{2}+z_{4}(A-\hat{A} \\
& \left.-F \operatorname{sgn}\left(z_{4}\right)\right) \leq 0
\end{aligned}
$$

\section{B. Lateral Dynamics}

In this subsection, the robust backstepping controller based on Lyapunov's method is designed for the lateral dynamics in the presence of wind gusts. Linear model equations of the lateral dynamics under external disturbance are rewritten 
as: $\dot{y}=v, \dot{v}=Y_{v} v+g \phi$,

$\dot{\phi}=p, \quad \dot{p}=L_{v} v+L_{p} p+L_{b} b_{1}+\delta$

where, $\delta$ is an unknown parameter which is estimated as $\hat{\delta}$. The estimation error on $\delta$ is assumed bounded by known constant $F_{l}$, that is, $|\delta-\hat{\delta}| \leq F_{l}$.

Step1: The design process starts with the definition of the lateral position tracking error i.e.,

$$
e_{1}=y-y_{d}, \quad \dot{e}_{1}=\dot{y}-\dot{y}_{d}, \dot{e}_{1}=v
$$

We consider $v$ as a virtual control input and define $v_{d}$ virtual control law for Eqn.(20). Let $e_{2}$ be an error variable representing the difference between the actual and virtual control inputs i.e.,

$$
e_{2}=v-v_{d}, v=e_{2}+v_{d},
$$

Therfore, $\dot{e}_{1}=e_{2}+v_{d}$

At this stage we would like to design a virtual control law $v_{d}$ which would make $e_{1} \rightarrow 0$ as $t \rightarrow \infty$. Consider a control Lyapunov function, $W_{1}=\frac{1}{2} e_{1}^{2}$

And its time derivative as follows,

$$
\dot{W}_{1}=e_{1} \dot{e}_{1}, \quad \dot{W}_{1}=e_{1}\left(e_{2}+v_{d}\right)
$$

We can now select an appropriate virtual control $v_{d}$ which would make $\dot{W}_{1} \leq 0$ A possible choice is $v_{d}=-c_{1} e_{1}$ then $W_{1}=-c_{1} e_{1}^{2}+e_{1} e_{2}$. Clearly if $e_{2}=0$ then $W_{1}=-c_{1} e_{1}^{2} \leq 0$

where, $c_{l}$ is a scalar parameter which can be used to tune the output response. Now consider the time derivative of $v_{d}$ as,

$$
\dot{v}_{d}=-c_{1} \dot{e}_{1}, \quad \dot{v}_{d}=-c_{1} v
$$

Step2: We derive the error dynamics for $e_{2}=v-v_{d}$ and its derivative as follows,

$$
\dot{e}_{2}=\dot{v}-\dot{v}_{d}, \dot{e}_{2}=Y_{v} v+g \phi+c_{1} \dot{e}_{1}, \quad \dot{e}_{2}=Y_{v} v+g \phi+c_{1} v
$$

In which $\phi$ is viewed as a virtual control input. Now define a virtual control law $\phi_{d}$ and let $e_{3}$ be an error variable representing the difference between the actual and virtual control inputs i.e., $e_{3}=\phi-\phi_{d}$

Therefore, $\dot{e}_{2}=\left(Y_{v}+c_{1}\right) v+g\left(e_{3}+\phi_{d}\right)$. Now choose a control Lyapunov function

$$
W_{2}=W_{1}+\frac{1}{2} e_{2}^{2}
$$

And its derivative as,

$$
\begin{gathered}
\dot{W}_{2}=\dot{W}_{1}+e_{2} \dot{e}_{2} \\
\dot{W}_{2}=-c_{1} e_{1}^{2}+e_{2}\left\{e_{1}+\left(Y_{v}+c_{1}\right) v+g \phi_{d}\right\}+g e_{2} e_{3}
\end{gathered}
$$

We can now select an appropriate virtual control $\phi_{d}$ to cancel out some terms related to $e_{1}, e_{2}$ and $v$, while the term involving $e_{3}$ cannot be removed.

$$
\phi_{d}=-g^{-1}\left\{e_{1}+\left(Y_{v}+c_{1}\right) v+c_{2} e_{2}\right\}
$$

Therefore, $\quad \dot{W}_{2}=-c_{1} e_{1}^{2}-c_{2} e_{2}^{2}+g e_{2} e_{3}$, clearly if $e_{3}=0$ then $\dot{W}_{2}=-c_{1} e_{1}^{2}-c_{2} e_{2}{ }^{2}$. Now the time derivative of $\phi_{d}$

$$
\begin{aligned}
& \dot{\phi}_{d}=-g^{-1}\left\{\dot{e}_{1}+\left(Y_{v}+c_{1}\right) \dot{v}+c_{2} \dot{e}_{2}\right\} \\
& \dot{\phi}_{d}=-g^{-1}\left\{v+\left(Y_{v}+c_{1}\right)\left(Y_{v} v+g \phi\right)+c_{2} \dot{e}_{2}\right\}
\end{aligned}
$$

Step3: We derive the error dynamics for $e_{3}=\phi-\phi_{d}$. And its derivative as follows,

$$
\begin{aligned}
\dot{e}_{3} & =\dot{\phi}-\dot{\phi}_{d} \\
\dot{e}_{3} & =p+g^{-1}\left\{v+\left(Y_{v}+c_{1}\right)\left(Y_{v} v+g \phi\right)\right\}+ \\
& g^{-1} c_{2}\left\{\left(Y_{v}+c_{1}\right) v+g\left(e_{3}+\phi_{d}\right)\right\}
\end{aligned}
$$

In which $p$ is viewed as a virtual control input. Now define a virtual control law $p_{d}$ and let $e_{4}$ be an error variable representing the difference between actual and virtual controls i.e.,

$$
\begin{gathered}
e_{4}=p-p_{d}, \quad p=e_{4}+p_{d} \\
\dot{e}_{3}=z_{4}+p_{d}+g^{-1}\left\{v+\left(Y_{v}+c_{1}\right)\left(Y_{v} v+g \phi\right)\right\}+ \\
g^{-1} c_{2}\left\{\left(Y_{v}+c_{1}\right) v+g\left(e_{3}+\phi_{d}\right)\right\}
\end{gathered}
$$

Now choose a control Lyapunov function

$$
W_{3}=W_{2}+\frac{1}{2} e_{3}^{2}
$$

And its derivative as,

$$
\begin{gathered}
\dot{W}_{3}=\dot{W}_{2}+e_{3} \dot{e}_{3} \\
\dot{W}_{3}=-c_{1} e_{1}^{2}-c_{2} e_{2}^{2}+g e_{3} e_{2}+ \\
e_{3}\left[e_{4}+p+g^{-1}\left\{v+\left(Y_{v}+c_{1}\right)\left(Y_{v} v+g \phi\right)\right\}+\right. \\
g^{-1} c_{2}\left\{\left(Y_{v}+c_{1}\right) v+g\left(e_{3}+\phi_{d}\right)\right]
\end{gathered}
$$

We can now select an appropriate virtual control $p_{d}$ to cancel some terms related to $e_{1}, e_{2}, e_{3}$ and $v$, while the term involving $e_{4}$ cannot be removed.

$$
\begin{aligned}
p_{d}= & -g e_{2}-g^{-1}\left\{v+\left(Y_{v}+c_{1}\right)\left(Y_{v} v+g \phi\right)\right\} \\
& -g^{-1} c_{2}\left\{\left(Y_{v}+c_{1}\right) v+g\left(e_{3}+\phi_{d}\right)\right]-c_{3} e_{3}
\end{aligned}
$$

And its derivative as follows,

$$
\begin{gathered}
\dot{p}_{d}=-g \dot{z}_{2}-g^{-1}\left\{\dot{v}+\left(Y_{v}+c_{1}\right)\left(Y_{v} \dot{v}+g \dot{\phi}\right)\right\} \\
-g^{-1} c_{2}\left\{\left(Y_{v}+c_{1}\right) \dot{v}+g\left(\dot{z}_{3}+\dot{\phi}_{d}\right)\right]-c_{3} \dot{z}_{3}
\end{gathered}
$$


Suppose, $\dot{p}_{d}=f\left(e_{2}, e_{3}, v, \phi, \phi_{d}\right)$

Therefore, $\dot{W}_{3}=-c_{1} e_{1}^{2}-c_{2} e_{2}^{2}-c_{3} e_{3}^{2}+e_{3} e_{4}$. Clearly if

$e_{4}=0$ then $\dot{W}_{3}=-c_{1} e_{1}^{2}-c_{2} e_{2}^{2}-c_{3} e_{3}^{2} \leq 0$

Step4: We derive the error dynamics for $e_{4}=p-p_{d}$ and its time derivative as,

$$
\begin{aligned}
& \dot{e}_{4}=\dot{p}-\dot{p}_{d} \\
& \dot{e}_{4}=L_{v} v+L_{p} p+L_{b} b_{1}+\delta-f\left(e_{2}, e_{3}, v, \phi, \phi_{d}\right)
\end{aligned}
$$

In the above equation (17) the actual control input appears. Our objective is to design the actual control input ' $b_{1}$ ' such that $e_{1}, e_{2}, e_{3}$, and $e_{4}$ converge to zero as $\mathrm{t} \rightarrow \infty$. Choose a Lyapunov function $W_{4}$ as, $W_{4}=W_{3}+\frac{1}{2} e_{4}{ }^{2}$ and its derivative as follows, $\dot{W}_{4}=\dot{W}_{3}+e_{4} \dot{e}_{4}$

$$
\begin{aligned}
& \dot{W}_{4}=-c_{1} e_{1}^{2}-c_{2} e_{2}^{2}-c_{3} e_{3}^{2}+e_{3} e_{4}+ \\
& e_{4}\left(L_{v} v+L_{p} p+L_{b} b_{1}+\delta-f\left(e_{2}, e_{3}, v, \phi, \phi_{d}\right)\right)
\end{aligned}
$$

We choose our control input $b_{1}$ as follows,

$$
\begin{aligned}
b_{1}=- & L^{-1}{ }_{b}\left(z_{3}+L_{v} v+L_{p} p+\hat{\delta}-\right. \\
& \left.f\left(e_{2}, e_{3}, v, \phi, \phi_{d}\right)+c_{4} e_{4}+F \operatorname{sgn}\left(e_{4}\right)\right)
\end{aligned}
$$

Then,

$$
\begin{gathered}
\dot{W}_{4}=-c_{1} e_{1}^{2}-c_{2} e_{2}^{2}-c_{3} e_{3}^{2}-c_{4} e_{4}^{2}+ \\
e_{4}\left(\delta-\hat{\delta}-F \operatorname{sgn}\left(e_{4}\right)\right) \leq 0
\end{gathered}
$$

\section{Vertical Dynamics}

In this subsection, a nonlinear robust backstepping controller for helicopter altitude stabilization in the presence of external wind gusts is designed based on Lyapunov function. The vertical dynamics is dependent on the vertical altitude $z$ and the collective pitch, $\theta_{0}$. We know the altitude $z$ is controlled by $T$ through collective pitch $\theta_{0}$. So, vertical dynamics motion equations of the helicopter are written as follows:

$$
\dot{z}=w
$$

$$
\dot{w}=\frac{m g-T}{m}+\varepsilon
$$

where, $\varepsilon$ is an unknown parameter but estimated as $\hat{\varepsilon}$. The estimation error on $\varepsilon$ is assumed bounded by known constant $F_{v}$, that is $|\varepsilon-\hat{\varepsilon}| \leq F_{v}$.

Step 1: The design process starts with the definition of the altitude tracking error and its derivative as follows:

$$
e_{1}=z-z_{0}, \dot{e}_{1}=\dot{z}, \dot{e}_{1}=w
$$

We view $w$ as a virtual control input for equation (14) and define $w_{d}$ is the virtual control law. Let $e_{2}$ be an error variable representing the difference between the actual and virtual control of (24) i.e., $e_{2}=w-w_{d}, w=e_{2}+w_{d}$

Therefore, $\dot{e}_{1}=e_{2}+w_{d}$

In this step our control objective is to design a virtual control law $w_{d}$ which would make $e_{1} \rightarrow 0$ as $t \rightarrow \infty$.

Now consider a control Lyapunov function, $V_{1}=\frac{1}{2} e_{1}^{2}$, whose time derivative as follows,

$$
\dot{V}_{1}=e_{1} \dot{e}_{1}, \quad \dot{V}_{1}=e_{1}\left(e_{2}+w_{d}\right)
$$

We can now select an appropriate virtual control $w_{d}$ which would make $\dot{V}_{1} \leq 0$.

$$
\text { A possible choice is } w_{d}=-\alpha e_{1}
$$

where, $\alpha$ is a scalar parameter which can be used to tune the output response.

Therefore, $\dot{V}_{1}=-\alpha e_{1}^{2}+e_{1} e_{2}$

Clearly if $e_{2}=0$, then $\dot{V}_{1}=-\alpha e_{1}^{2} \leq 0$

Now time derivative of the equation (24) as follows,

$$
\dot{w}_{\dot{\alpha}}=-\alpha \dot{e}_{1}, \dot{w}_{\dot{\alpha}}=-\alpha w
$$

$$
\dot{e}_{2}=\dot{w}-\dot{w}_{d},
$$

$$
\dot{e}_{2}=g-\frac{T}{m}+\varepsilon+\alpha w
$$

Again we know,

$$
T=\frac{\rho a(\Omega R)^{2} A_{b}}{2}\left[\frac{\theta_{0}}{3}\left(1+\frac{3}{2} \mu^{2}\right)-\frac{1}{2} \lambda^{\prime}\right]
$$

where,

$$
\begin{aligned}
& \lambda^{\prime}=\frac{v_{i}+v_{n}}{\Omega R}, \mu=\frac{v_{t}}{\Omega R}, v_{n}=\left(a_{1}+i_{s}\right) u-b_{1} v-v_{z}, \\
& v_{t}^{2}=u^{2}+v^{2}, \quad v_{i}^{2}=\sqrt{\left(\frac{\hat{v}}{2}\right)^{2}+\left(\frac{T}{2 \rho A}\right)^{2}}-\frac{\hat{v}^{2}}{2} \\
& \hat{v}=\sqrt{v_{t}^{2}+\left(v_{n}+v_{t}\right)^{2}} .
\end{aligned}
$$

Now, by substituting the value of $T$ into Eqn. (27) we get,

$$
\dot{e}_{2}=g-\frac{B \theta_{0}}{3 m}\left(1+\frac{3}{2} \mu^{2}\right)+\frac{B}{2 m} \lambda^{\prime}+\varepsilon+\alpha w
$$

where,

$$
B=\frac{\rho a(\Omega R)^{2} A_{b}}{2}
$$


In the above equation the actual control input appears. Our objective is to design the actual control input $\theta_{0}$ such that $e_{1}$ and $e_{2}$ converge to zero. Now choose a Lyapunov function $V_{2}$ as follows,

$$
V_{2}=V_{1}+\frac{1}{2} e_{2}^{2}
$$

And its time derivative as follows,

$$
\begin{gathered}
\dot{V}_{2}=\dot{V}_{1}+e_{2} \dot{e}_{2} \\
\dot{V}_{2}=-\alpha e_{1}^{2}+e_{1} e_{2}+e_{2}\left\{g-\frac{B \theta_{0}}{3 m}\left(1+\frac{3}{2} \mu^{2}\right)+\frac{B}{2 m} \lambda^{\prime}+\varepsilon+\alpha w\right\} \\
\dot{V}_{2}=-\alpha e_{1}^{2}+e_{2}\left\{e_{1}+g-\frac{B \theta_{0}}{3 m}\left(1+\frac{3}{2} \mu^{2}\right)+\frac{B}{2 m} \lambda^{\prime}+\varepsilon+\alpha w\right\}
\end{gathered}
$$

We choose our control input $\theta_{0}$ as follows,

$$
\theta_{0}=\frac{3 m}{B\left(1+\frac{3}{2} \mu^{2}\right)}\left[\begin{array}{l}
e_{1}+g+\frac{B}{2 m} \lambda^{\prime}+\hat{\varepsilon}+\alpha w \\
+\beta e_{2}+F_{v} \operatorname{sgn}\left(e_{2}\right)
\end{array}\right]
$$

Then

$$
\begin{gathered}
\dot{V}_{2}=-\alpha e_{1}^{2}+e_{2}\left\{e_{1}+g-\left(e_{1}+g+\frac{B}{2 m} \lambda^{\prime}+\right.\right. \\
\left.\left.\alpha w+\hat{\varepsilon}+\beta e_{2}+F_{v} \operatorname{sgn}\left(e_{2}\right)\right)+\frac{B}{2 m} \lambda^{\prime}+\varepsilon+\alpha w\right\} \\
\dot{V}_{2}=-\alpha e_{1}^{2}-\beta e_{2}^{2}+e_{2}\left(\varepsilon-\hat{\varepsilon}-F_{v} \operatorname{sgn}\left(e_{2}\right)\right) \leq 0
\end{gathered}
$$

\section{LQR Controller Design}

Linear quadratic regulator (LQR) controller is considered as one of the most important state space based optimal control methods. In LQ problem, the system dynamics are described by a set of linear differential equations and its cost is described by a quadratic function. The solution for the problem is provided by the linear-quadratic regulator. LQR theory results in a linear control law which minimizes the integral over an infinite time interval of the weighted sum of the squares of the elements of the system state and control vectors. Consider the linear time-invariant system

$$
\begin{aligned}
& \dot{x}=A x+B u \\
& y=C x
\end{aligned}
$$

wherein the state $x \in \mathfrak{R}^{n}$, the input control $u \in \mathfrak{R}^{m}$, and the measured output $y \in \mathfrak{R}^{p}$. To design the LQR controller, the first step is to select the weighting matrices $\mathrm{Q}$ and $\mathrm{R}$, where $\mathrm{Q}$ is weighting factors that weight the states and $\mathrm{R}$ is also weighting factors that weights inputs. Then the feedback $\mathrm{K}$ can be computed and the closed loop system responses can be found by simulation. The sate feedback control law has the form:

$$
u=-K x
$$

where $K$ is a $m \times n$ matrix which minimizes the following performance matrix:

$$
J=\int_{0}^{\infty}\left(x^{T} Q x+u^{T} R u\right) d t
$$

In (32), $Q$ and $R$ are the positive definite (or positive semi-definite) weighting matrices which will balance the relative importance of the input and state in the cost function $J$ that we are trying to optimize. The state feedback gain can be computed as follows,

$$
K=R^{-1} B^{T} P
$$

where $P$ is a positive definite matrix obtained from the solution of the following algebraic Riccati equation:

$$
A^{T} P+P A+Q-P B R^{-1} B^{T} P=0
$$

\section{Simulation Results}

In this section, robust backstepping control and LQR are now compared via simulations. Simulation is conducted for the case in which the initial positions are set to $x=0 \mathrm{~m}, \mathrm{y}=0$ $\mathrm{m}$ and $\mathrm{z}=-2 \mathrm{~m}$ and its desired positions to $\mathrm{x}=0 \mathrm{~m}, \mathrm{y}=0 \mathrm{~m}$ and $z=2 \mathrm{~m}$. The roll angle trim $\phi_{\text {ref }}$ is initialized at $4.5^{\circ}$ to compensate for the tail rotor thrust. The effectiveness of the proposed controller is investigated in a gusty environment by comparing it with the LQR controller. In order to determine $F, F_{l}$ and $F_{v}$ at first we set up a mean velocity of the wind gusts and found the pitch rate, roll rate and vertical acceleration response. Again we set up another mean velocity of the wind gusts and found the pitch rate, roll rate and vertical acceleration response. From these two results we found the pitch rate, roll rate vertical acceleration variation due to the external wind gusts. From these pitch rat, roll rate and vertical acceleration variation we set up the bound constants are to be $F=0.5 \mathrm{deg} / \mathrm{s}, F_{l}=0.4 \mathrm{deg} / \mathrm{s}$ and $F_{v}=0.8$ $\mathrm{m} / \mathrm{s}^{2}$ on the basis of pitch rate, roll rate vertical acceleration variation respectively for external wind gusts. The weighting matrices for both longitudinal and lateral modes are considered to be $Q_{\text {lon }}=\operatorname{diag}\left[\begin{array}{llll}0.1 & 0.1 & 0.5 & 0.6\end{array}\right], R_{\text {lon }}=30$ and $Q_{\text {lat }}=\left[\begin{array}{llll}0.5 & 0.01 & 0.5 & 0.6\end{array}\right], R_{\text {lat }}=80$ respectively. The state feedback matrices for both longitudinal and lateral dynamics are $K_{\text {lon }}=\left[\begin{array}{llll}-0.1421 & 0.1043 & 0.6046 & -0.1414\end{array}\right]$ and $K_{\text {lat }}=\left[\begin{array}{ll}0.1169 \\ 0.00\end{array}\right.$ $0.06200 .44690 .0866]$. The difference between two controls appears in fig. 1 where tracking errors in $x, y$ and $z$ are less by using backstepping controller than LQR. 

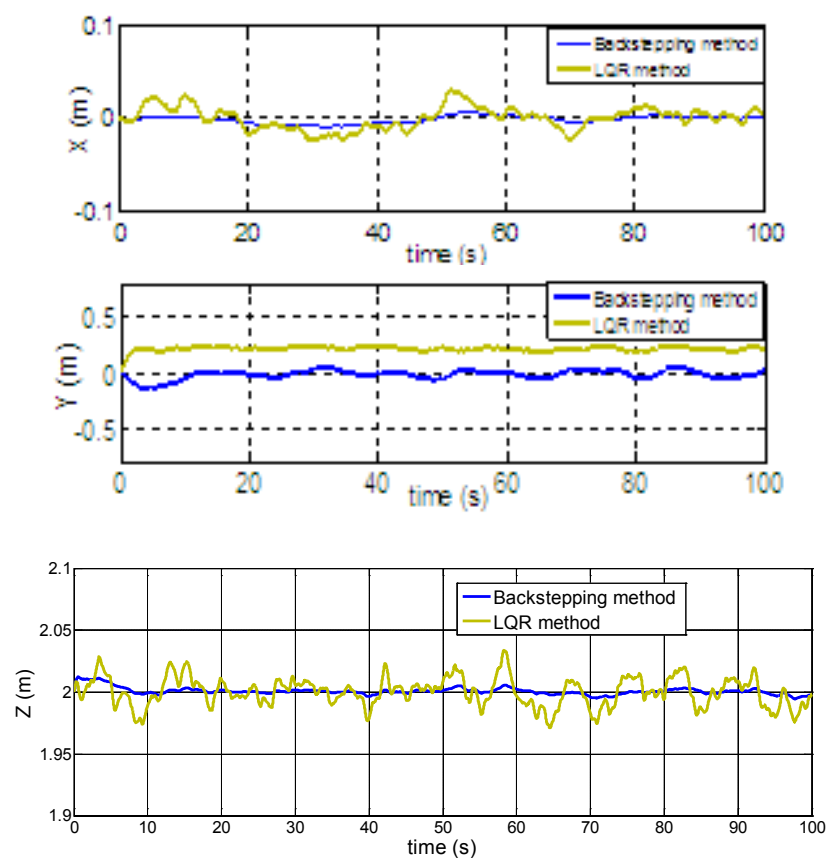

Figure 1. Helicopter position response using backstepping and LQR Controller

Fig. 1 shows the performances of the proposed robust backstepping controller (blue line) and LQR controller (green line) and it is clear from the simulation results that the position tracking error is almost zero for robust backstepping controller but relatively large for the LQR controller.
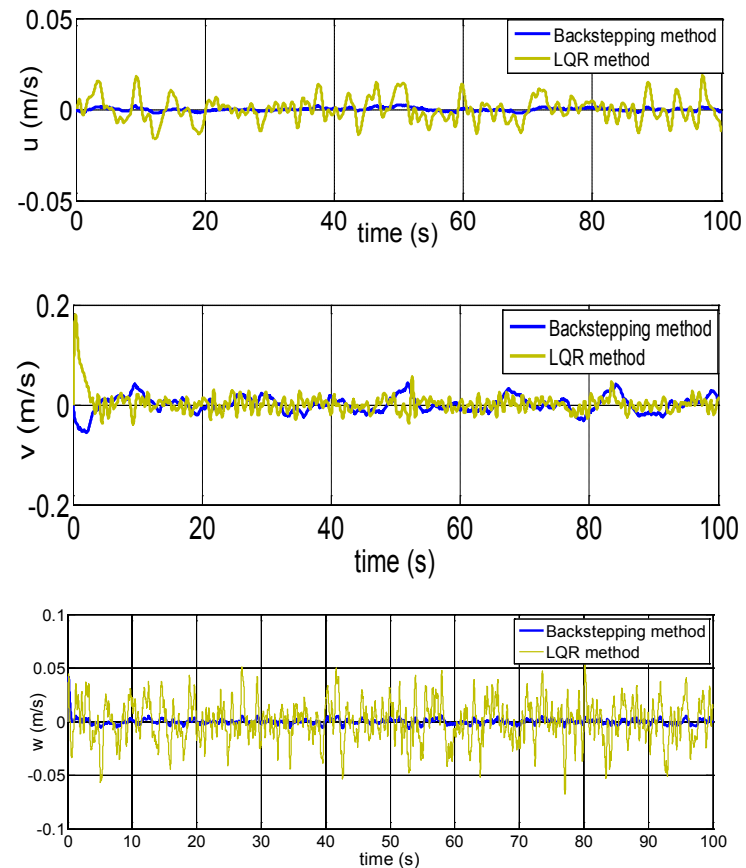

Figure 2. Helicopter velocity response using backstepping and LQR controller
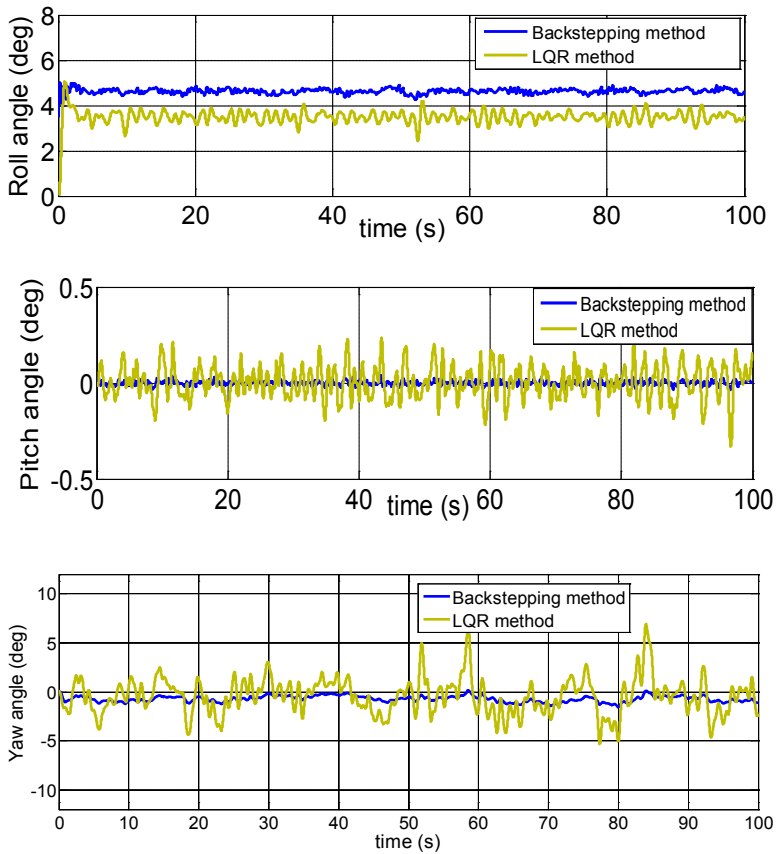

Figure 3. Helicopter orientation response using backstepping and LQR controller

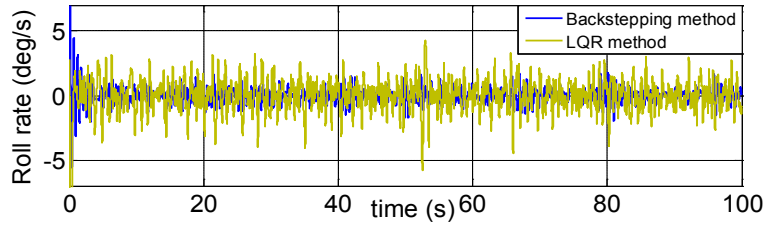

Figure 4. Helicopter roll rate response using backstepping and LQR controller

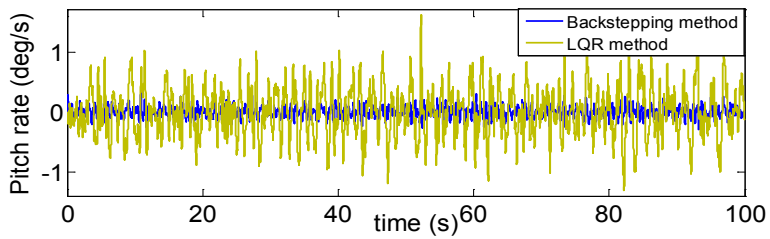

Figure 5. Helicopter pitch rate response using backstepping and LQR controller

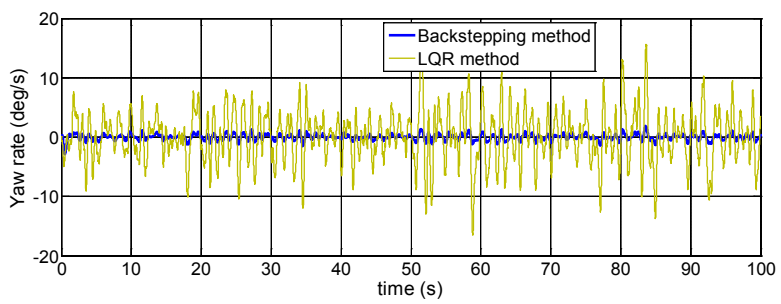

Figure 6. Helicopter yaw rate response using backstepping and $L Q R$ controller 

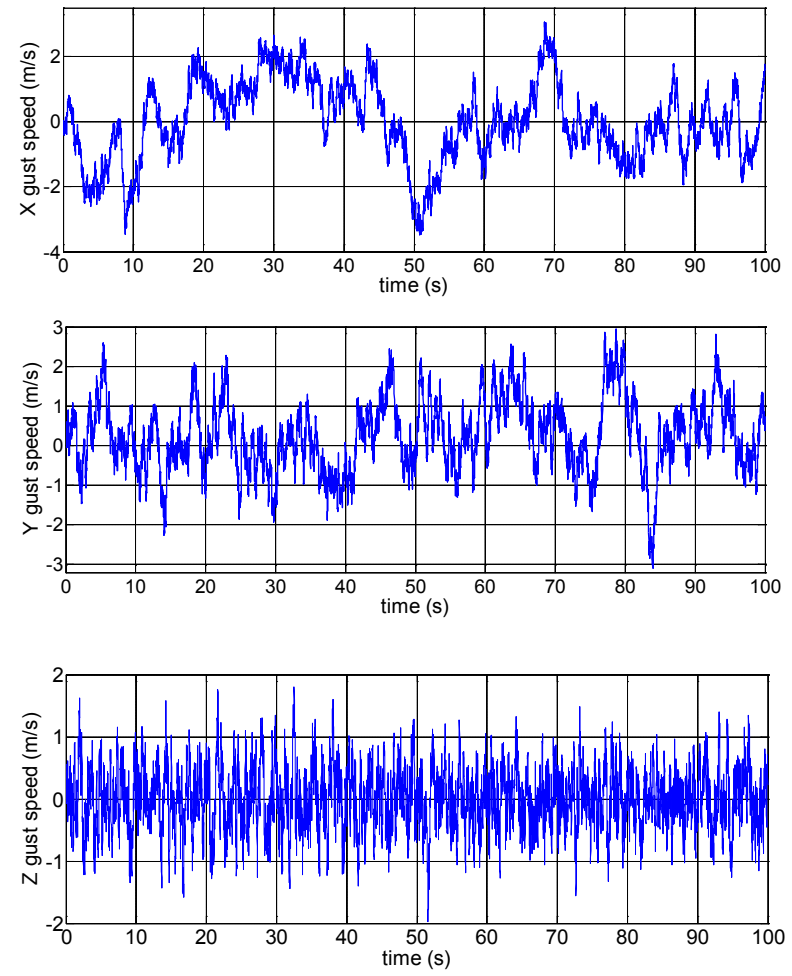

Figure 7. Wind gusts to test controller

The faster responses are the outcome of the rapid velocity responses depicted in Fig.2 and it is clear that velocities are converged quickly to zero with smaller excursions and smaller fluctuation unlike LQR controller. The roll, pitch and yaw angles of both controllers are presented in Fig. 3. For the robust backstepping controller, the roll angle settles approximately within the desired value $\left(4.5^{0}\right)$, but for the LQR controller, it settles in the range of $2.5^{\circ}$ to $3.95^{\circ}$. Fig. 4 , Fig. 5, and Fig.6, shows the roll, pitch and yaw rates of the small Vario XLC helicopter which are almost zero for the robust backstepping controller while the oscillations are not damped completely for the LQR controller. Therefore it is clear that the proposed robust backstepping controller is better than the LQR controller in a gusty environment. The Dryden wind disturbance is shown in fig.7 that used in the simulation to test the controllers.

\section{Conclusion}

In this paper, a Lyapunov's based backstepping control and LQR control have been applied for the control of longitudinal, lateral and vertical position of the small scale helicopter in the presence of wind gusts. Comparison simulation results show that the robust backstepping controller can settle the longitudinal, lateral and vertical position of the small helicopter more rapidly than an LQR controller in a gusty environment. Considering the simulation performed, LQR controller also successful to stabilize the longitudinal, lateral and vertical position of the helicopter but as seen by the fact that helicopter cannot hover at the desired position ( $\mathrm{x}=0 \mathrm{~m}, \mathrm{y}=0 \mathrm{~m}$ and $\mathrm{z}=2 \mathrm{~m}$ ). Even for large external disturbances, the proposed backstepping controller is robust against this external disturbance. So, our propose backstepping controller better than LQR controller in a gusty environment. But we can say that the two proposed controls algorithm produces satisfactory performances. Implementation of our proposed autonomous flight control method on the real system and flight test to prove its feasibility in real applications are remained for future work.

\section{REFERENCES}

[1] D.J.Wallcer, M.C. Turner, AJ. Smerlas, ME. Strange, and A.W. Gubbels, "Robust Control of the Longitudinal and Lateral Dynamics of the BELL 205 Helicopter," Proceedings of the American Control Conference San Diego, California June 1999.

[2] Kumeresan A. Danapalasingam, John-Josef Leth, Anders la Cour-Harbo and Morten Bisgaard "Robust Helicopter Stabilization in the Face of Wind Disturbance" 49th IEEE Conference on Decision and Control, December 15-17, 2010 Hilton Atlanta Hotel, Atlanta, GA, USA.

[3] T. Cheviron, F. Plestan, and A. Chriette, "A robust guidance and control scheme of an autonomous scale helicopter in presence of wind gusts," International Journal of Control, Vol. 82, No. 12 December 2009, 2206-2220.

[4] Xilin Yang, Matt Garratt, and Hemanshu Pota, “ A Nonlinear Position Controller Operations of Rotary-wing UAVs," 18th IFAC World Congress Milano (Italy) August 28- September 2, 2011.

[5] T. Cheviron, F. Plestan, and A. Chriette, "A robust guidance and control scheme of an autonomous scale helicopter in presence of wind gusts," International Journal of Control, Vol. 82, No. 12 December 2009, 2206-2220.

[6] Ioannis A. Raptis and Kimon P. Valavanis, "Velocity and Heading Tracking Control For Small-Scale Unmanned Helicopters" American Control Conference on O'Farrell Street, San Francisco, CA, USA June 29 - July 01, 2011.

[7] D. McLean. Automatic Flight Control Systems. Prentice Hall, 1990

[8] M. Garratt. Biologically inspired vision and control for an autonomous flying vehicle. $\mathrm{PhD}$ thesis, Austarlian National University, October 2007.

[9] T. Cheviron, F. Plestan and A. Chriette,"A robust guidance and control scheme of an autonomous scale helicopter in presence of wind gusts", International Journal of Control, Vol. 82, No. 12, December 2009, 2206-2220.

[10] L. Guo, C. Melhuish, and Q. Zhu, "Towards neural adaptive hovering control of helicopters," in Proc. IEEE Int. Conf. Control Applications, Glasgow, U.K., Sep. 2002 , pp. 54-58.

[11] N. C. Nigam and S. Narayanan, "Application of Radom Vibration”, Springer-Verlag, New Delhi, 1994.

[12] S. Suresh, P. Kashyab and M. Nabi, “Automatic Take-off Control System for Helicopter - An Ho Apporach,” 11th 
Conf. Control, Automation, Robotics and Vision, Singapore, 7-10th December, 2010.

[13] Alberto Isidori, Lorenzo Marconi and Andrea Serrani, "Robust Nonlinear Motion Control of a Helicopter" IEEE Transactions on Automatic Control, Vol. 48, No. 3 March 2003.

[14] L. Guo, C. Melhuish, and Q. Zhu, "Towards neural adaptive hovering control of helicopters",in Proc. IEEE Int. Conf. Control Applications, Glasgow, U.K., Sep. 2002 , pp. 54-58.

[15] T. K. Roy, M. Garrat, H. R. Potat and H. Teimoori, "Robust Backstepping Control for Longitudinal and Lateral Dynamics of Small Scale Helicopter," Journal of University of Science and Technology of China, Vol. 42, No. 7, Jul. 2012

[16] T. K. Roy, "Robust backstepping control for small helicopter" Master thesis, November 2012, The University of New South Wales, Australia.

[17] M. Tischler and R. Remple, "Aircraft and rotorcraft system identification: engineering methods with flight-test examples," in AIAA, 2006.

[18] H. Teimoori, H. R. Pota, M. Garratt, and M. K. Samal, "Planar trajectory tracking controller for a small-sized helicopter considering servos and delay constraints," in 37th Annual Conference on IEEE Industrial Electronics Society, November 2011, pp. 681-686.

[19] C. Castillo, W. Moreno, and K. Valavanis, "Unmanned helicopter waypoint trajectory tracking using model predictive control," in Mediterranean Conference on Control Automation, June 2007, pp. 1-8.
[20] I. Raptis and K. Valavanis, "Velocity and heading tracking control for small-scale unmanned helicopters," in American Control Conference (ACC), July 2011, pp. 1579-1586.

[21] S. A. Budiyonoa, "Optimal tracking controller design for a small-scale heli-copter," in Journal of Bionic Engineering, 2007, pp. 271-280.

[22] G. Cai, B. Chen, K. Peng, M. Dong, and T. Lee, "Modeling and control system design for a UAV helicopter," in 14th Mediterranean Conference on Control and Automation, June 2006, pp. 1-6.

[23] M. Garratt, B. Ahmed, and H. Pota, "Platform enhancements and system identification for control of an unmanned helicopter," in 9th International Conference on Control, Automation, Robotics and Vision (ICARCV), December 2006, pp. 1-6.

[24] Z. Bai, P. Liu, J. Wang, X. Hu, and X. Zhao, "Control system design of a small-scale unmanned helicopter," in 8th IEEE International Conference Control and Automation (ICCA), June 2010, pp. 1414-1417.

[25] D. Song, J. Qi, L. Dai, J. Han, and G. Liu, "Modeling a small-size unmanned helicopter using optimal estimation in the frequency domain," in 15th International Conference on Mechatronics and Machine Vision in Practice, December 2008, pp. 97-102.

[26] H. Degang, W. Jiande, F. Yugang, W. Xiaodong, and F. Ting, "Modeling of miniature unmanned helicopter for hovering status based on least square support vector machine," in 3rd International Symposium on Knowledge Acquisition and Modeling (KAM), October 2010, pp. 114-116. 